\title{
| COMPOSITION DU GROUPE DE TRAVAIL
}

Animateur

Georges PÉDRO

\section{Rapporteur}

Bernard BLANZAT

\section{Membres du groupe}

Pierre ALBRECHT

Jacques BERTHELIN

Daniel COSSA

Isabelle FEIX

Robert GUILLAUMONT
Correspondant de l'Académie des sciences - Directeur de recherche émérite à l'Institut national de la recherche agronomique (Inra)

Directeur de recherche au Centre national de la recherche scientifique (CNRS) - Chargé de mission à l'Académie des sciences

Directeur de recherche au Centre national de la recherche scientifique (CNRS)

- Directeur de l'unité mixte de recherche "Substances naturelles, évolution, réactivité » (UMR 7509 CNRS)

Directeur de recherche émérite au Centre national de la recherche scientifique (CNRS)

- Laboratoire des interactions, minéraux, matières organiques dans les sols (Limos) (CNRS UMR 7137 et université Henri Poincaré)

Biogéochimiste à l'Institut français de recherche pour l'exploitation de la mer (Ifremer) - Centre de Nantes

Chef du département Gestion biologique et sols à l'Agence de l'environnement et de la maîtrise de l'énergie (Ademe)

Membre de l'Académie des sciences Professeur honoraire à l'université Paris-Sud Orsay 
Alain Yves HUC

Patrick LAVELLE

Michel LEBRUN

Yves LUCAS

Henri MÉTIVIER

Guy OURISSON

Patrick RAIMBAULT

Jacques RANGER

Claudine SCHMIDT-LAINÉ
Professeur à l'Institut français du pétrole (IFP)

Correspondant de Y'Académie des sciences - Professeur à l'université Pierre-et-MarieCurie - Directeur du Laboratoire d'écologie des sols tropicaux (UMR 137 IRD ef universités Paris VI, VII, XII)

Unité mixte de recherche Biochimie et physiologie moléculaire des plantes (UMR 5004 CNRS, UM2, Inra et Ensam)

Directeur du Laboratoire Processus de transferts et d'échanges dans l'environnement (Protee) - Unité de recherche EA 3819 université du Sud Toulon Var

Directeur de recherche honoraire au Commissariat à l'énergie atomique Professeur honoraire à l'Institut national des sciences et techniques nucléaires

Membre de l'Académie des sciences Professeur émérite à l'université Louis Pasteur

Directeur de recherche au CNRS Laboratoire d'océanographie et de biogéochimie (UMR 6535 CNRS et université de la Méditerranée)

Directeur de l'unité de recherche Biogéochimie des écosystèmes forestiers (UR 1138 Inra)

Directeur scientifique à l'Institut de recherche pour l'ingénierie de l'agriculture et de l'environnement (Cemagref) - Antony

\section{Coordonnateur}

Jean DERCOURT

Secrétaire perpétuel de l'Académie des sciences - Professeur émérite à l'université Pierre-et-Marie-Curie 


\section{Ont également participé à la rédaction du rapport}

Chapitre 1

Jérôme GAILLARDET

Chapitre 3

Jacques BERTHELIN

Jean-Pierre BOUDOT

Guilhem BOURRIÉ

Frédéric GÉRARD

Jean Dominique MEUNIER

Fabienne TROLARD

\section{Chapitre 5.1}

Marc BENOÎT

Nathalie BRÉDA
Directeur de l'UFR des sciences de la Terre, de l'environnement et des planètes (STEP) Institut de physique du globe de Paris

Directeur de recherche émérite au Centre national de la recherche scientifique (CNRS) - Laboratoire des interactions minéraux, matières organiques dans les sols (Limos) (CNRS UMR 7137 et université Henri Poincaré)

Laboratoire des interactions minéraux, matières organiques dans les sols (Limos) (UMR 7137 CNRS et université Henri Poincaré)

Unité de recherche Géochimie des sols et des eaux, Inra - Europôle méditérranéen de l'Arbois

Unité de recherche Biogéochimie des écosystèmes forestiers (UR 1138 Inra) - Nancy

Centre européen de recherche et d'enseignement des géosciences de l'environnement (Cerege) (UMR 6635 CNRS) - Europôle méditerranéen de l'Arbois

Unité de recherche Géochimie des sols et des eaux, Inra - Europôle méditérranéen de l'Arbois

Inra - Station Sad Mirecourt - Nancy

Unité mixte de recherche Écologie et écophysiologie forestières de Nancy (UMR A1 137 Inra et université Henri Poincaré) 
Étienne DAMBRINE

Jean-Luc DUPOUEY

Alain FRANC

Jean GARBAYE

Frédéric GÉRARD

André GRANIER

Guy LANDMANN

Denis LOUSTAU

Francis MARTIN

Chapitre 6.1

José MARTINEZ

Chapitre 6.2

Philippe CROCHON
Unité de recherche Biogéochimie

des écosystèmes forestiers - Inra Nancy

Unité mixte de recherche Écologie

et écophysiologie forestières (UMR A1 137

Inra et université Henri Poincaré) - Nancy

Unité mixte de recherche Biodiversité, gènes et écosystèmes (UMR 1202 Inra et université Bordeaux I)

Unité mixte de recherche Interactions arbres/micro- organismes (UMR 1136 Inra et université Henri Poincaré) - Inra Nancy

Unité de recherche Biogéochimie des écosystèmes forestiers (UR 1138 Inra) Inra Nancy

Unité mixte de recherche Écologie et écophysiologie forestière - Inra Nancy

Directeur-adjoint - Gip Ecofor

Unité de recherche Écologie fonctionnelle et physique de l'environnement (UR 1263 Inra) - Inra Bordeaux

Unité mixte de recherche Interactions arbres/micro- organismes (UMR 1136 Inra et université Henri Poincaré) - Inra Nancy

Directeur de recherche - Chef de l'unité de recherche Gestion environnementale et traitement biologique des déchets - Institut de recherche pour l'ingénierie de l'agriculture et de l'environnement (Cemagref) - Rennes 
Jean-Didier GAY

IRSN - Direction de la Sûreté des usines, des laboratoires, des transports et des déchets (DSU) - Service de Sûreté des déchets radioactifs (SSD) - Bureau d'évaluation des barrières de confinement des stockages de déchets radioactifs (BECS) -

Fontenay-aux-Roses

Henri MÉTIVIER

Directeur de recherche honoraire au Commissariat à l'énergie atomique Professeur honoraire à l'Institut national des sciences et techniques nucléaires

Jean-Marc PÉRÈS

IRSN - Direction de l'Environnement et de l'intervention (DEI) - Service d'Analyse des risques liés à la géosphère (SARG) Fontenay-aux-Roses

Chapitre 7

Christian TAMPONNET

Chapitre 8

Francis ANDREUX

Colette MUNIER-LAMY

Chapitre 9

Marie-Hélène TUSSEAUVUILLEMIN
IRSN - Direction de l'Environnement et de l'intervention (DEI) - Service d'Études du comportement des radionucléides dans les écosystèmes (SECRE) - Cadarache

Unité mixte de recherche Microbiologie et géochimie des sols (UMR 1229 Inra et université de Bourgogne)

Laboratoire des interactions minéraux, matières organiques dans les sols (Limos) (UMR 7137 CNRS et université Henri Poincaré)

Unité Hydrosystèmes et bioprocédés à l'Institut de recherche pour l'ingénierie de l'agriculture et de l'environnement (Cemagref) - Antony 
Chapitre 10.3

Évelyne BARKER

Patrick BOUISSET

Pierre GERMAIN

Olivier MASSON

IRSN - Direction de l'Environnement et de l'intervention (DEI) - Service de Traitement des échantillons et de métrologie pour l'environnement (STEME) - Laboratoire de Mesure de la radioactivité dans l'environnement Orsay

IRSN - Direction de l'Environnement et de I'intervention (DEI) - Service de Traitement des échantillons et de métrologie pour l'environnement (STEME) - Laboratoire de Mesure de la radioactivité dans l'environnement Orsay

IRSN - Direction de l'Environnement et de I'intervention (DEI) - Service d'Études du comportement des radionucléides dans les écosystèmes (SECRE) - Cherbourg-Octeville

IRSN - Direction de l'Environnement et de I'intervention (DEI) - Service d'Études du comportement des radionucléides dans les écosystèmes (SECRE) - Laboratoire de Radioécologie de Cherbourg-Octeville

\section{Auteurs des annexes du chapitre 4}

Abdesselam ABDELOUAS

Erich ANSOBORLO

Bernd GRAMBOW

Remo CHIAPPINI
Maître-assistant à École des mines de Nantes - Laboratoire Subatech (UMR 6457 IN2P3/CNRS et université de Nantes)

Direction de l'Énergie nucléaire - CEA

Professeur à l'École des mines de Nantes Laboratoire Subatech (UMR 6457 IN2P3/CNRS et université de Nantes)

Direction des Applications militaires Chef de service Radioanalyse chimie environnement - CEA 
Ryzsard LOBINSKI

Gilles MONTAVON

Christophe MOULIN

Valérie MOULIN
Directeur de recherche - Laboratoire de Chimie analytique, bio-informatique et environnement (UMR 5034 CNRS)

Chargé de recherche au CNRS Laboratoire Subatech (UMR 6457 IN2P3/CNRS et université de Nantes)

Direction de l'Énergie nucléaire - Chef du laboratoire Spéciation des radionucléides et molécules - CEA

Direction de l'Énergie nucléaire - Chef du programme Domaine maîtrise des risques CEA

\section{Auteurs de la note du chapitre 7}

Alain Yves HUC

Bernard OLLIVIER

Franck HAESELER

Daniel PRIEUR

Michel MAGOT
Professeur à l'Institut français du pétrole (IFP)

Direcleur adjoint de l'unité mixte de recherche Microbiologie et biotechnologie des environnements chauds (UMR 180 IRD/universités Aix-Marseille 1 et 2)

Chef du département de Géochimie - Institut français du pétrole (IFP)

Professeur de microbiologie - Laboratoire de Microbiologie des environnements extrêmes (UMR 6197) Université de Bretagne occidentale

Professeur à l'Université de Pau et des pays de l'Adour - Laboratoire d'Écologie moléculaire - Institur de biologie environnementale Aquitaine Sud (IBEAS) 


\section{Auteurs des encadrés}

Chapitre 5.2

Dominique BOUST

Sabine CHARMASSON

Présentation deuxième partie

Roland POSS

Chapitre 6.2

Catherine GRIMALDI

Michel GRIMALDI

Chapitre 8

Caroline de MALET

Chapitre 9

Pascal BAILLY du BOIS
IRSN - Direction de l'Environnement et de l'intervention (DEI) - Service d'Études du comportement des radionucléides dans les écosystèmes (SECRE) - Cherbourg-Octeville

IRSN - Direction de l'Environnement et de l'intervention (DEI) - Service d'Étude et de surveillance de la radioactivité dans I'environnement (SESURE) - Laboratoire d'Études radioécologiques en milieu continental et marin (LERCM) - La Seyne-sur-Mer

Directeur de recherche - Unité de recherche Solutions - IRD Montpellier

Unité mixte de recherche Sol, agronomie, spatialisation - Inra Rennes

Unité mixte de recherche Biodiversité et fonctionnement du sol - IRD Bondy

Journaliste « Le Figaro »

IRSN - Direction de l'Environnement ef de l'intervention (DEI) - Service d'Études du comportement des radionucléides dans les écosystèmes (SECRE) - Cherbourg-Octeville 